Results 104 patients were randomized. In the efficacy evaluable population, $42 \%$ of XmAb5871-treated subjects reached Day 225 without LOI vs $28.6 \%$ of the placebo group $(\mathrm{p}=0.18)$ with $40.4 \%$ vs $23.1 \%(\mathrm{p}=0.06)$ achieving this endpoint in the ITT population. In those with LOI, no (0\%) XmAb5871 patients vs 9 (30\%) placebo had SLEDAI increase 7 with $3(13 \%)$ vs 7 (23\%) developing BILAG A scores. Six XmAb5871-treated patients were withdrawn for infusion-related events. The efficacy evaluable population excluded 10 placebo patients vs 2 XmAb5871 for other reasons, increasing placebo response proportions compared to the ITT population. Time to LOI was significantly longer in XmAb5871-treated patients than placebo $(p=0.025$, see figure 1$)$.

The most common AEs in XmAb5871-treated patients were transient, infusion-related gastrointestinal side effects during the 1 st or 2 nd infusion. There were 8 SAEs in $7 \mathrm{XmAb}-$ treated subjects, 5 in 4 placebo patients, no opportunistic infections, and no deaths. Infection rate was low compared to other SLE trials.

Conclusions Results from this small trial, designed to maximize interpretability, supports further evaluation of XmAb5871 in SLE.

Funding Source(s): This study was funded by Xencor Inc.

\section{SENSITIVITY OF AN AUTOMATED FLUORESCENCE ENZYME IMMUNOASSAY VERSUS IMMUNOFLUORESCENCE AMONGST PATIENTS WITH SYSTEMIC LUPUS ERYTHEMATOSUS: A SYSTEMATIC LITERATURE REVIEW AND META-ANALYSIS}

${ }^{1}$ Carmen Andalucia*, ${ }^{1}$ Sigrid Sjölander, ${ }^{2}$ Michelle Orme. ${ }^{1}$ Thermo Fisher Scientific; ${ }^{2}$ ICERA Consulting Ltd

\subsection{6/lupus-2019-Ism.99}

Background Antinuclear antibodies (ANA) play an important role in the diagnosis and classification of Systemic Lupus Erythematosus (SLE). Positivity of ANA by immunofluorescence (IIF) or an equivalent assay is part of the 1997 American College of Rheumatology (ACR) revised classification criteria and the 2012 revised criteria for the diagnosis of SLE from the Systemic Lupus International Collaborating Clinics (SLICC).

The aim of this study was to provide an overview of the sensitivity of automated fluorescence immunoassay (FEIA) versus IIF in SLE patients and consequently the number of false negatives.

Methods MEDLINE, EMBASE and Cochrane database searches (2000-March 2018) were conducted to identify fully-paired, cross-sectional or case-control studies of the diagnostic accuracy of FEIA and IIF in connective tissue diseases.

A meta-analysis of the sensitivity of FEIA versus IIF in SLE patients was conducted using hierarchical, mixed-effect models.

Methodological quality of included studies was assessed using the QUADAS-2 tool.

Results Out of 1798 papers identified, 11 studies (691 SLE patients) met the criteria for inclusion and reported the diagnostic accuracy of both IIF and FEIA. Four of the studies included consecutive patients (single-gate) and seven studies were case-control (two-gate).
Overall, sensitivity was $81 \%$ (95\% CI 74\%-86\%) for FEIA and $88 \%(95 \%$ CI $79 \%-93 \%)$ for IIF, the difference being not statistically significant $(\mathrm{p}=0.14)$.

In the QUADAS-2 assessment, four studies included a positive ANA test as criteria for the diagnosis/classification of SLE which could introduce a high risk of bias in the overall sensitivity estimate. After excluding these four studies, the sensitivity point estimate for the remaining eight studies (431 patients) was $82 \%$ for FEIA and $81 \%$ for IIF $(\mathrm{p}=0.9)$.

Based on a $1.51 \%$ average prevalence of SLE in the singlegate studies, for every 1000 consecutive suspected patients screened, 15 patients will have SLE. Three out of fifteen will have a false negative test with FEIA versus two out of fifteen with IIF when sensitivity estimates from all studies are used. Three out of fifteen will have a false negative test with both tests when sensitivity estimates exclude the four studies that incorporated ANA results into the patient classification/ diagnosis.

Conclusions Pooled sensitivity estimates of IIF and the automated FEIA in SLE patients was similar and not statistically different.

Taking into consideration the average prevalence of SLE in the single gate studies, both tests showed similar number of false negatives.

Funding Source(s): Carmen Andalucia and Sigrid Sjölander are Thermo Fisher Scientific employees. Michelle Orme was a Thermo Fisher Scientific consultant.

\section{DIFFERING OPINIONS ON CLINICAL RESEARCH BETWEEN HEALTHCARE PROVIDERS AND LUPUS PATIENTS}

${ }^{1}$ Cristina Arriens*, ${ }^{2}$ Dylan Forciea, ${ }^{1}$ Fredonna Carthen, ${ }^{1}$ Dallas McCance, ${ }^{1} J$ Judith A James, ${ }^{1}$ Joan T Merrill. 'OKlahoma Medical Research Foundation; ${ }^{2}$ Oseberg

10.1136/lupus-2019-Ism.100

Background Although systemic lupus erythematosus (SLE) disproportionately affects minority racial groups, they are significantly under-represented in clinical trials. This produces underpowered conclusions in race-based sub-group analyses. The decision to participate in clinical research is complex. Primary care providers (PCPs) have the ability to introduce the idea of clinical trials and to refer to specialists who participate in clinical trials. We evaluated SLE knowledge and implicit bias in clinical research participation in both PCPs and lupus patients.

Methods Lupus patients and PCPs completed a pre-test consisting of knowledge and belief questions followed by an educational program about lupus, clinical research, and human subjects protections. The same questions were repeated as part of the post-test. Responses to knowledge questions were analyzed by Fishers exact test for between group (patients vs. PCPs) comparisons or McNemars test for within group (pretest vs. post-test) comparisons. Belief questions were scored on a Likert scale and analyzed by Mann-Whitney or Wilcoxon matched pairs for between group and within group comparisons. 
A

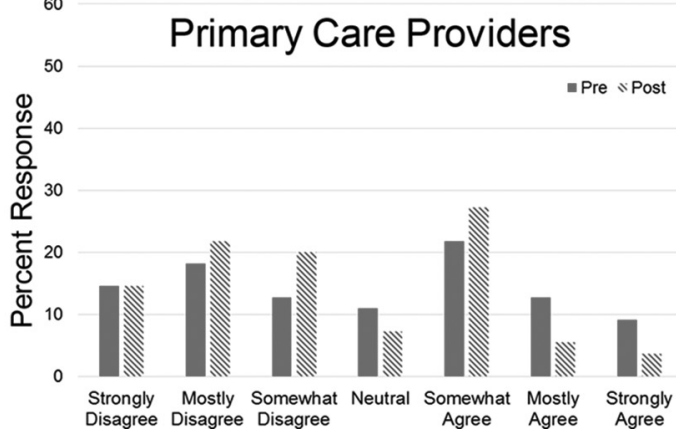

B

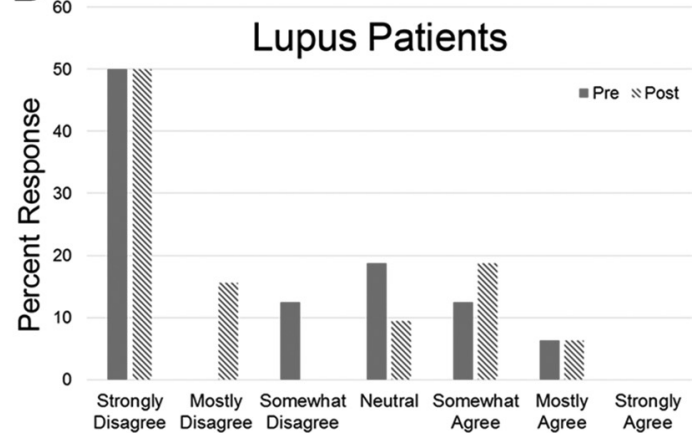

Abstract 100 Figure 1 Perceived Impact of Poverty and Study Protocol Adherence. A) Primary care providers were asked to respond to the following statement Indigent patients are less likely to comply with a study protocol before and after the educational program and had a shift towards increased disagreement (median [interquartile range], Pre $4[2,5]$ and Post $3[2,5], p=0.024)$ ). B) Similarly lupus patients were asked to respond to I think people who are poor or have less education are less likely to correctly follow the instructions they are given during a research study with a majority of responses disagreeing which remained stable after the program (Pre 2 [1, 4] and Post 1.5 [1, 4.75], $p=0.72$ ). Significant differences in responses between providers and patients were noted both before the program $(p=0.0037)$ and after the program $(p=0.0088)$. The shift in providers response towards disagreement after the program was not large enough to result in post-test responses similar to the patients.

Results 55 providers and 32 lupus patients completed the questionnaires and educational program. Knowledge topics included 1) triggers of SLE, 2) lupus racial differences, and 3) informed consent. There was a statistically significant difference between PCPs and patients on the pre-test informed consent topic (PCP 46\% correct and patient 15\%, p=0.0005). Post-test results were similar between groups. The education program resulted in improvement in knowledge scores for PCPs (Q1 $42 \%$ to $85 \%, \quad \mathrm{p}<0.001$; Q2 $56 \%$ to $91 \%$, $\mathrm{p}<0.0001 ; \mathrm{Q} 384 \%$ to $87 \%, \mathrm{p}=0.5$ ) and patients (Q1 $38 \%$ to $84 \%, \mathrm{p}=0.0003$; Q2 $56 \%$ to $75 \%, \mathrm{p}=0.1$; Q3 $47 \%$ to $72 \% \mathrm{p}=0.01)$. Topics of clinical trial belief questions included perceived risks of trials, differing racial background between provider and patient, education level needed for trial participation, and perceived effect of poverty on protocol compliance. The final topic demonstrated the greatest incongruence between PCPs and patients (figure 1).

Conclusions Beliefs about race, education, and poverty may impact referral to clinical trial centers and clinical trial participation. More than a third of PCPs believe that indigent people are poor candidates for clinical trials. Despite a shift away from this attitude following an educational program, providers remained much more likely than lupus patients to hold this opinion.

Funding Source(s): HHS-OMH-CPI-MP-17-002-1

\section{D-MANNOSE AMELIORATES SYSTEMIC LUPUS ERYTHEMATOSUS-LIKE DISEASE IN B6.MRL/LPR MOUSE MODEL}

${ }^{1}$ Haiting Wang*, ${ }^{2}$ Xiangyu Teng, ${ }^{2}$ Georges Abboud, ${ }^{2}$ Wei Li, ${ }^{2}$ Laurence Morel. ${ }^{1}$ Renji Hospital South Campus, Shanghai JiaoTong University School of Medicine, Shanghai, China; ${ }^{2}$ Department of Pathology, Immunology, and Laboratory Medicine, University of Florida, Gainesville, FL, USA

\subsection{6/lupus-2019-Ism.101}

Background Systemic lupus erythematosus (SLE) is an autoimmune disease that is characterized by high morbidity and mortality and its treatment remains challenging. Inflammatory dendritic cells (DCs) have been shown to participate in the initiation and perpetuation of lupus pathogenesis, and tolerogenic DCs have a potential for cell-based therapy in this condition. The mannose receptor (MR, CD206) is a C-type lectin expressed by DCs and its cross-linking induces anti-inflammatory immunosuppressive effects. D-Mannose is a C-2 epimer of glucose that exhibit immunoregulatory effect in models of autoimmune diseases, such as type 1 diabetes and lung airway inflammation. However, the function of D-Mannose treatment in lupus remains unknown.

Methods B6.MRL-Fas(lpr) (B6.lpr) mice at 4 months of age were treated with D-Mannose in drinking-water for 2 months. Autoantibody production and immune cell activation were compared between the two groups. In vitro, GMCSF bone marrow-derived dendritic cells (BMDCs) from non-autoimmune B6 mice were cultured for 5 days to generate mature dendritic cells. On day 5, BMDCs were treated with $10 \mathrm{mM}$ glucose (G10) or $10 \mathrm{mM}$ Mannose (M10) for 24 hour and pulsed with LPS for an additional 4 hour. Surface markers and cytokines secretion of BMDCs were analyzed.

Results The D-Mannose treatment significantly decreased serum anti-dsDNA antibody at week 4. It also increased the percentage of naïve $\mathrm{T}(\mathrm{Tn})$ cells and decreased CD4 $+\mathrm{T}$ cell activation measured as CD44 +expression. Follicular helper T (TFH) cells/follicular regulatory $\mathrm{T}$ (TFR) cells ratio was reduced after D-Mannose treatment. The low frequency of regulatory $\mathrm{T}$ (Treg) cells in $\mathrm{B} 6 / \mathrm{lpr}$ mice was also expanded after treatment. Besides, D-Mannose treatment increased CD206 expression on spleenic DCs. In vitro experiments showed that D-Mannose promoted a tolerogenic phenotype in BMDCs by decreasing the expression of activation markers (CD40, CD80, CD86) and promoting that of inhibitory markers (CD206 and CD64) expression in B6 mice. Additionally, D-Mannose reduced inflammatory cytokine secretion in BMDCs.

Conclusions D-Mannose ameliorates the development of lupuslike disease in the B6/lpr mouse model, which may be due to the induction of tolerogenic DCs.

Funding Source(s): National Key R and D Program of China (2017YFC0909000) 